\title{
Minimal Supersymmetric CPN Models
}

\author{
K.J. Barnes \\ Department of Physics \& Astronomy, \\ University of Southampton \\ Southampton, SO17 1BJ \\ United Kingdom \\ Telephone: +44 1703592097 \\ Email: kjb@phys.soton.ac.uk
}

\begin{abstract}
Supersymmetric CPN models based on underlying bosonic Kahler manifolds have not been thought to arise directly from constrained linear ones. A counterexample for $N=4$ is presented using improved understanding of membranes in superstring theories leading to crucial central terms modifying the algebra of supercharge densities. The example has an immediate extension to all higher $N$.
\end{abstract}

PACS codes: 11.10 Lm, 11.25.-W, 11.30.Pb, 11.30.Rd

Keywords: supersymmetry, Kahler, superstrings, membranes, central terms. 
It has recently been established that $C P 2$ can be realised as a non-linear supersymmetric model as the result of constraining a linear supersymmetric model [1]. Massless Goldstone bosons arise from components of global symmetries which are spontaneously broken. There is no extra symmetry for Goldstone bosons in supersymmetry. Instead the supersymmetry forces complexification of scalars. This leads to an increased number of massless excitations in general, with complete doubling of the original number in some cases. Despite previously believed theorems to the contrary by Lerche [2] and Shore [3], the $C P 2$ case was established as a counterexample. The key contribution leading to this possibility was that of Hughes and Polchinski [4], which showed that the original anticommutator for supersymmetric charges had to be generalised to include a central term at the underlying current density level. This is a direct result of the more modern viewpoint that supermembranes are just as fundamental as elementary particles in string theory. The key point seems to be that this is a case where the symmetry of the hamiltonian is larger than the symmetry of the $S$-matrix. When the anticommutator algebra for supersymmetic charges is generalised to local form as

$$
\partial_{\mu} T\left(j_{A \alpha}^{\mu}(x) \bar{j}_{B \dot{\beta}}^{\nu}(y)\right)=2\left(\sigma^{\rho}\right)_{\alpha \dot{\beta}} T_{\rho}^{\nu} \delta^{4}(x-y) \delta_{A B}+2\left(\sigma^{\nu}\right)_{\alpha \dot{\beta}} C_{A B} \delta^{4}(x-y)
$$

the appearance of the central terms $C_{A B}$ is crucial. The authors take advantage of the fact that $T^{\mu \nu}$ is not the only unique conserved symmetric tensor since $T^{\mu \nu}+C \eta^{\mu \nu}$ is also conserved. Thus equation (1) is clearly finite and Lorentz invariant, and from it follow the usual consequences of degenerate multiplets for unbroken supersymmetries and Goldstone fermions for those that are broken. In momentum space, with $C_{A B}$ diagonal and $<T^{\mu \nu}>=\Lambda \eta^{\mu \nu}$, this gives

$$
q_{\mu}<j_{A \alpha}^{\mu}(q) \bar{j}_{A \dot{\beta}}^{\nu}>=2\left(\sigma^{\nu}\right)_{\alpha \dot{\beta}}\left(\Lambda+C_{A A}\right)+0(q)
$$

where there is no sum over $A$. For those $A$ such that $\Lambda+C_{A A} \neq 0$, equation (2) implies a $1 / \not q$ singularity in the two current correlation; $j_{A \alpha}^{\mu}$ couples the vacuum to a massless 
fermion with coupling strength $\left[2\left(\Lambda+C_{A A}\right)\right]^{1 / 2}$, where $\Lambda+C_{A A} \geq 0$. It is now clear how to evade the extra unwanted Goldstone bosons where the underlying coset manifold is indeed Kahler. The crucial point of extending the underlying algebra of supercharge current densities by central terms has to be combined not merely with a Kahler $G / H$, but that manifold has to be reexpressed as a quotient of the complexified $G$ (denoted as $G^{C}$ ) by a maximally extended complexification of $H$ (denoted $\hat{H}$ ). By following the elegant treatment of Itoh, Kugor and Kunitoma [5], this method will display an explicit mapping manifesting the homeomorphism between $G / H$ and $G^{C} / \hat{H}$. Since the bosonic coset space for $C P 4$ is

$$
\frac{G}{H}=\frac{S U_{3}}{S U_{2} \times U_{1}}
$$

a convenient starting point for the appropriate notation is given by the original GellMann matrices [6]. Note that $\lambda_{8}$ and $\lambda_{3}$ are in the Cartan subalgebra, and that the raising operators are $E_{1}=1 / 2\left(\lambda_{1}+i \lambda_{2}\right), E_{2}=1 / 2\left(\lambda_{4}+i \lambda_{5}\right)$ and $E_{3}=1 / 2\left(\lambda_{6}+i \lambda_{7}\right)$, with $E_{-1}=E_{1}^{\dagger}, E_{-2}=E_{2}^{\dagger}$ and $E_{-3}=E_{3}^{\dagger}$ as the lowering operators. It is clear that all the raising and lowering operators are nilpotent in this representation. This feature obviously extends to larger $N$ and ensures that constructing the Kahler potential is essentially immediate in all cases. Following reference [5] a projection operator $\eta$ with its only entry a one in the bottom right hand corner is defined by

$$
\eta=\frac{1}{3} 1-\sqrt{\frac{1}{3}} \lambda_{8}
$$

and the complex subgroup $\hat{H}$ specified by the relationship

$$
\hat{h} \eta=\eta \hat{h} \eta
$$

This implies that the generators of $\hat{H}$ are $\lambda_{8}, \lambda_{3}, E_{1}, E_{-1}, E_{-2}$ and $E_{-3}$, and that $E_{2}$ and $E_{3}$ are the four elements of the algebra spanning $G^{c} / \hat{H}$.

Extending the notation of reference [1], the original (unconstrained) supersymmetric action is constructed from nine (complex) chiral superfields. In components, 
with

$$
y^{m}=x^{m}+i \theta \sigma^{m} \bar{\theta},
$$

these have the form

$$
\begin{aligned}
\Phi(x, \theta, \bar{\theta}) & =\phi(y)+\sqrt{2} \theta \lambda_{\phi}(y)+\theta^{2} F_{\phi}(y), \\
\Sigma_{8}(x, \theta, \bar{\theta}) & =\sigma_{8}(y)+\sqrt{2} \theta \lambda_{8}(y)+\theta^{2} F_{8}(y), \\
\Sigma_{3}(x, \theta, \bar{\theta}) & =\sigma_{3}(y)+\sqrt{2} \theta \lambda_{3}(y)+\theta^{2} F_{3}(y), \\
\Delta_{A}(x, \theta, \bar{\theta}) & =\Delta_{A}(y)+\sqrt{2} \theta \Lambda_{A}(y)+\theta^{2} F_{A}(y),
\end{aligned}
$$

where $A=(-1,1)$,

$$
\begin{aligned}
\Delta_{-2}(x, \theta, \bar{\theta}) & =\delta_{-2}(y)+\sqrt{2} \theta \Lambda_{-2}(y)+\theta^{2} F_{\Delta}(y), \\
\Delta_{-3}(x, \theta, \bar{\theta}) & =\delta_{-3}(y)+\sqrt{2} \theta \Lambda_{-3}(y)+\theta^{2} F_{-3}(y), \\
\Gamma_{\mu}(x, \theta, \bar{\theta}) & =\gamma_{\mu}(y)+\sqrt{2} \theta \Omega_{\mu}(y)+\theta^{2} F_{\mu}^{\Gamma}(y),
\end{aligned}
$$

where $\mu=(2,3), \sigma^{m}\left(-1, \tau^{a}\right)$, and the $\tau^{a}$ are the Pauli matrices $(a=1,2,3)$. The chiral superfields transform under $S U_{3}$ as indicated by the index structure, including $\Phi$ which is a singlet. The most general supersymmetric action is then written as

$$
\begin{gathered}
I=\int d^{8} z\left[\bar{\Phi} \Phi+\bar{\Sigma}_{8} \Sigma_{8}+\bar{\Sigma}_{3} \Sigma_{3}+\bar{\Delta}_{A} \Delta_{A}+\bar{\Delta}_{-2} \Delta_{-2}+\bar{\Delta}_{-3} \Delta_{-3}+\bar{\Gamma}_{\mu} \Gamma_{\mu}\right] \\
+\int d^{6} s W+\int d^{6} \bar{s} \bar{W}
\end{gathered}
$$

where the superpotential $W$ is a functional of chiral superfields only. Combining the eight non-singlet $S_{3}$ superfields with their respective matrices into the matrix

$$
M=\Sigma_{8} \lambda_{8}+\ldots+\Gamma_{\mu} E_{-\mu},
$$

reveals that, under chiral $S U_{3} \times S U_{3}, M$ transforms as

$$
M \rightarrow L M R^{\dagger},
$$

where the $\gamma_{5}$ structure is suppressed, and taking

$$
W=k \Phi \operatorname{det} M,
$$


where $k$ is a constant, ensures that the model reduces to the usual bosonic (Kahler) model below the symmetry breaking scale. This starting action now yields the potential

$$
\begin{gathered}
V=F_{\phi} \bar{F}_{\phi}+F_{8} \bar{F}_{8}+F_{3} \bar{F}_{3}+F_{A} \bar{F}_{A}+F_{-2} \bar{F}_{-2}+F_{-3} \bar{F}_{-3}+F_{\mu}^{\Gamma} \bar{F}_{\mu}^{\Gamma} \\
=4 k^{2} \phi \bar{\phi}\left[\sigma_{8} \bar{\sigma}_{8}+\sigma_{3} \bar{\sigma}_{3}+\delta_{A} \bar{\delta}_{A}+\delta_{-2} \bar{\delta}_{-2}+\delta_{-3} \bar{\delta}_{-3}+\gamma_{\mu} \bar{\gamma}_{\mu}\right] \\
+k^{2}\left[\sigma_{8}^{2}+\sigma_{3}^{2}+\delta_{A} \delta_{A}+\gamma_{3} \delta_{3}+\gamma_{\mu} \gamma_{A+2}\right]\left[\bar{\sigma}_{8}^{2}+\bar{\sigma}_{3}^{2}+\bar{\delta}_{A} \bar{\delta}_{A}+\bar{\gamma}_{3} \bar{\delta}_{3}+\bar{\gamma}_{\mu} \bar{\gamma}_{A+2}\right]
\end{gathered}
$$

In the formal limit as $k \rightarrow \infty$, the action becomes

$$
I=\int d^{8} z \frac{\Gamma_{\mu} \bar{\Gamma}_{\mu}}{4}
$$

as the constraints are satisfied by the superfield conditions

$$
\Sigma_{8}=\Sigma_{3}=\Delta_{A}=\Delta_{3}=\Gamma_{3}=0
$$

The superfield $\Phi$ can again be ignored as a non-interacting spectator. Notice that the pair of complex superfields $\Gamma_{\mu}$ are all that remain in the action, and they are not constrained.

In this notation the complex coset space is written in the form [5]

$$
L=\exp \left(\frac{-i}{2} \gamma_{2} E_{2}\right) \exp \left(\frac{-i}{2} \gamma_{3} E_{3}\right)
$$

and this gives an explicit mapping of the homeomorphism between $G / H$ and $G^{c} / \hat{H}$. Following references [5] the Kahler potential is given by

$$
K=\ln \operatorname{det}_{\eta}\left[\exp \left(\frac{-i \bar{\gamma}_{3} \bar{E}_{3}}{2}\right) \exp \left(\frac{-i \bar{\gamma}_{2} \bar{E}_{2}}{2}\right) \exp \left(\frac{-i \gamma_{2} E_{2}}{2}\right) \exp \left(\frac{-i \gamma_{3} E_{3}}{2}\right)\right] \text {, }
$$

where the notation indicates that the determinant is to be taken in the bottom right hand of the matrix in this representation. This reveals at once that

$$
K=\ln \left[1+\frac{\gamma_{\mu} \bar{\gamma}_{\mu}}{4}\right]
$$

which is the desired result. Notice how this presentation deals with the main objections which arose when it was claimed in reference [1] that the generalisation directly 
to $\mathrm{CPN}$ was possible. It is not necessary to find special co-ordinates for the manifold in order to demonstrate that it is Kahler. The $C P N$ manifolds are already known to be Kahler. It is time that having a general co-ordinate system in the $C P 2$ case was very useful from a descriptive viewpoint, but it is now clear that it was not really needed. Of course it was very convenient to use the nilpotency of $\tau^{+}$and $\tau^{-}$in the $C P 2$ case, but far from being restricted to that case it is now obvious that the number of nilpotent matrices rises with $N$. Finally, there was the well established feature that there is an increasing number of Kahler potentials with rising rank of $G$, and each introduces an extra arbitrary constant. Of course this current presentation just gives one particular combination, but as is always the case with counterexamples one is sufficient.

The author is grateful to Professor D.A.Ross for raising his interest in this type of work. This work is partly supported by PPARC grant number GR/L56329.

\section{References}

[1] K.J. Barnes, "Avoiding the theorem of Lerche and Shore", hep-th/9906212, submitted for publication.

[2] W. Lerche, Nucl. Phys. B238 (1984) 582.

[3] A.C.W. Kotcheff and G.M. Shore, Int. J. of Mod. Phys. A4 (1989) 4381.

[4] J. Hughes and J. Polchinski, Nucl. Phys B278 (1986) 147.

[5] K. Itoh, T. Kugo and H. Kunitomo, Nucl. Phys. 263 (1986) 295.

[6] M. Gell-man and Y. Ne'eman, The Eightfold Way (W.A. Benjamin Inc., New York, Amsterdam (1964)) p11. 